\title{
OPTIMUM LAUNCHING OF ELECTRON-CYCLOTRON \\ POWER FOR LOCALIZED CURRENT DRIVE IN A HOT TOKAMAK
}

Gary R. Smith

This paper was prepared for submittal to Radio Frequency Power in Plasmas Conference Irvine, California, May 1-3, 1989

May 1989

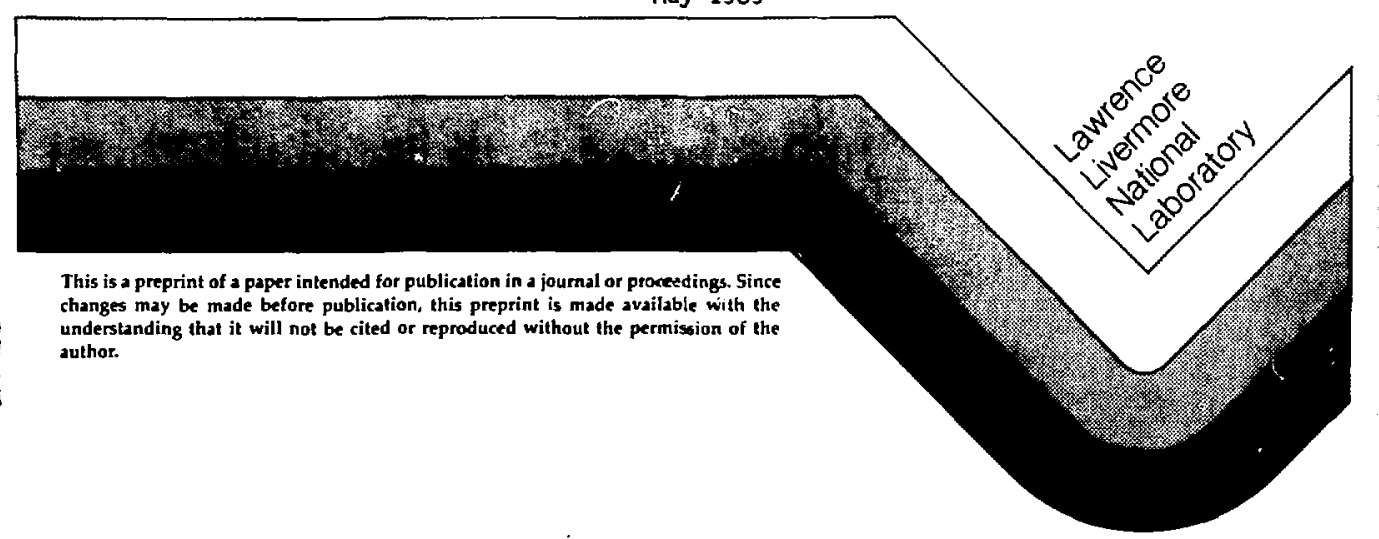


DISCLAMER

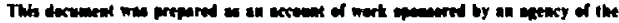

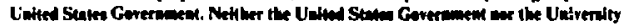

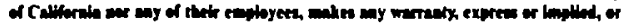

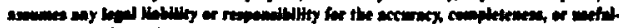

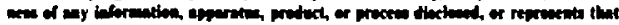

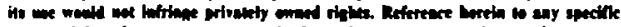

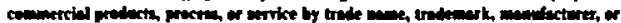

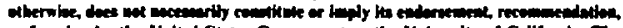

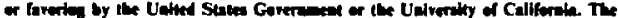

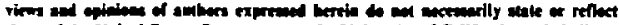

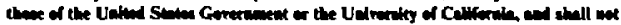

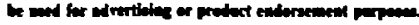


Optimum Launching of Electron-Cyclotron Power for Localized Current Drive in a Hot Tokamak

Gary R. Smith

Lawrence Livermore National Laboratory

University of California

Livermore, CA 94550

\begin{abstract}
Optimum launch parameters are determined for localized electron-cyclotron current drive near the magnetic axis and the $q=\mathbf{2}$ surface by solving several minimization problems. For central current drive, equatorial and bottom launch are compared. Localized current drive near $q=\mathbf{2}$ is studied for equatorial launch and for an alternative outside launch geometry that may be better for suppressing tearing modes and controlling disruptions.
\end{abstract}

\title{
JNTRODUCTION
}

One of the best uses of electron-cyclotron power in a tokamak is for localized current drive, because superior localizability tends to compensate for lower efficiency compared to other current-drive schemes. Localisation near the magnetic axis and the $q=1$ and $q=2$ surfaces is of interest. To determine launch scenarios that yield an optimum combination of efficiency and localizability ${ }_{1}$ one must search through a parameter space that includes the polojdal launch location, the launch direction, and the ratio $\Omega_{a x i s} / \omega$ of on-axis cyclotron frequency to wave frequency. This paper determines optimum launch parameters automatically by solving several minimuzation problems. Application is made to the ITER tokamak.

\section{PLASMA MODEL}

We use flux surfaces computed at Livermore for the JTER Basic Engineering Device during the technology phase. The specific case "j023" corresponds to the start of burn. At the nominal major radius of $R_{0}=5.5 \mathrm{~m}$, the magnetic field is $B_{0}=5.3 \mathrm{~T}$. The magnetic axis is at $R_{\text {axis }}=5.76 \mathrm{~m}$, where the field is $B_{\text {axis }}=5.08 \mathrm{~T}$. The safety factor $q$ increases monotonically from qaxir $=1.14$ at the magnetic axis to $995=3.64$ at $\psi=0.95$. The poloidal flux $\psi$ is normalized so that $\psi=0$ at the axis and $\psi=1$ at the separatrix. The $q=2$ surface is located at $\psi=\psi_{2}=0.725$.

The density and temperature profiles are not consistent with the pressure in the flux-surface calculation but have the widely used forms $n(\psi)=\bar{n}(1-\psi)^{\alpha_{n}}$ and $T_{e}(\psi)=\hat{T}_{c}(1-\psi)^{\alpha_{T}}$ with $T_{1}=T_{e}$ and $\alpha_{n}=0.5$ and $\alpha_{T}=1$. The peak (on-axis) density and temperature are $\hat{n}=10^{20} \mathrm{~m}^{-3}$ and $\hat{T}_{e}=35 \mathrm{keV}$.

Ray tracing and absorption is based on the weakly relativistic dielectric tensor of Shkarofsky. ${ }^{1}$ Current-drive efficiency is calculated with the inclusion of trapped-particle effects ${ }^{2}$ but with the omission of momentum transfer from hot to bulk electrons. ${ }^{3}$ The latter effect enhences current-drive efficiency by roughly $50 \%$ near the magnetic axis but much less on outer flux surfaces. Current-drive efficiency is reduced from that found in a pure plasma by our choice of $Z_{\text {eff }}=2.2$. In studying all physical situations, we employ single-ray calculations to understand the absorption and current-drive physics, then we complete the study with multi-ray calculations to learn about the sensitivities to finite size and divergence of realistic microwave beams.

\section{FORMULATION OF OPTIMIZATION CALCULATIONS}

Optimization calculations are performed with the aid of IMSL routine NOONF, which is based on work by Schittkowski. This routine attempts to minimize an objective function $F(x)$, 
subject to constraints and lower and upper bounds on the variables $x$. In this work we use no constreints but specify bounds to limit the parameter space searched by the solver. Depending on the current-drive problem of interest we make various choices of $F$ and $x$.

To maximize the current driven near the magnetic axis, we choose $F(x)=-I_{d}\left(\psi_{c}\right)$ with $\psi_{c}=0.1$, where $J_{d}(\psi)$ is the current driven on all flux surfaces between the axis $(\psi=0)$ and $\psi$. We model power launched along the equatorial plane $(Z=0)$ by a single ray, and we choose $x$ to consist of the microwave frequency $f$ and the angle $\phi_{\ell}$ between the launch direction and a line joining the launch point $(X=750 \mathrm{~cm}, Y=Z=0)$ with the machine center $\left(X=\mathrm{Y}^{*}=Z=0\right)$; symbolically, $x=\left\{f, \phi_{\ell}\right\}$. For power launched below the plasma ("bottom launch"), mare variables are required to specify the launch location and direction. Using a cylindrical coordinace system $(R, \Phi, Z)$, we fix the radial and azirnuthal components of the launch location at $R_{t}=830 \mathrm{~cm}$ and $\Phi_{c}=9.25^{\circ}$ and vary the vertical component of the launch location $Z_{\ell}$ and all components $\left(R_{t}, \Phi_{t}, Z_{t}\right)$ of a target location that determines the launch direction. Thus, we use $x=\left\{f, Z_{\ell}, R_{t}, \Phi_{\ell}, Z_{t}\right\}$.

Current drive just inside of the $q=2$ surface is capable, at least theoretically, of suppressing tearing modes, which may help to reduce the number or severity of disruptions. The current required drops dramatically es localization improves, ${ }^{5}$ so we choose to maximize $d I_{d} / d \psi$ just inside $\psi=\psi_{2}$. For power launched along the equatorial plane, we use $x=\left\{f_{1} \phi_{l}\right\}$ es before. An alternative launch geometry fixes the launch location below the equatorial plane at $(X=750 \mathrm{~cm}, Y=0, Z=-100 \mathrm{~cm})$ and varies two launch angles $\theta_{l}$ and $\phi_{\ell}$, which are related to the wavevector components at the launch location by $\tan \theta_{\ell}=-k_{Z} /\left(k_{X}^{2}+k_{1}^{2}\right)^{1 / 2}$ and $\tan \phi_{\ell}=k_{Y} / k_{X}$. Thus, we use $x=\left\{f, \theta_{\ell}, \phi_{\ell}\right\}$.

\section{OPTIMUM LAUNCH PARAMETERS FOR ITER}

Power launched along the equatorial plane drives maximum current near the magnetic axis for $f=198 \mathrm{GHz}$ and $\phi_{\ell}=39^{\circ}$. In terms of dimensionless paraineters that play important roles in the absorption and current-drive physics, this result can be expressed as $\Omega_{\text {axis }} / \omega=0.72$ and, at the absorption peak, $N_{1}=0.82$. These optimum parameters are similar to those found in Ref. 6 . However, that work found a substantially higher current-drive efficiency than we do at the optimum launch parameters, for reasons that are not presently understood. Taking momentum transfer into account (which Ref. 6 did not), we find a total driven current per incident watt of $I_{d} / P_{i}=0.05 \mathrm{~A} / \mathrm{W}$, or $\eta \equiv\langle n\rangle I_{d} R_{\text {axis }} / P_{i}=0.20 \times 10^{20} \mathrm{~A} / \mathrm{Wm}^{2}$.

Bottom launch is optimum for central current drive at $f=204 \mathrm{GHz}$ and with launch parameters $Z_{\ell}=-324 \mathrm{~cm}, R_{t}=557 \mathrm{~cm}, \Phi_{t}=-48^{\circ}$, and $Z_{\mathrm{t}}=100 \mathrm{~cm}$. A projection of the ray trajectory into the poloidal plane is shown in Fig. 1 . There is little refraction for this ray. The apparent curvature seen in Fig. 1 is mostly an effect of projection onto the non-Cartesian $R Z$ plane. The wave frequency corresponds to $\Omega_{a x i r} / \omega=0.7$ and, at the absorption peak, $N_{n}=0.82$. Although the bottom-launch geometry deposits power on electrons with higher mean energy than does launch in the equatorial plane, current-drive efficiency is only slightly better: $\eta=0.21 \times 10^{20} \mathrm{~A} / \mathrm{Wm}^{2}$. The single-ray bottom-launch calculation shows that over $90 \%$ of the driven current is concentrated within the flux surface $\psi=0.05$, which compares with $\psi=0.03$ for equatorial launch.

Power launched along the equalorial plane with the optimal $f=137 \mathrm{GHz}$ and $\phi_{\ell}=25^{\circ}$ drives a localized current with peak $d I_{d} / d \psi$ at $\psi=0.675$. The total driven current, ignoring the small momentum-transfer effect on this outer flux surface, is $0.022 \mathrm{~A} / \mathrm{W}$, which corresponds to $\eta=0.09 \times 10^{20} \mathrm{~A} / \mathrm{Wm}^{2}$. To characterize the localization of the driven current, we quote the width $\Delta \psi=0.15$ that contains the middle $80 \%$ of the current.

The alternative launch geometry for localized current drive near $q=2$ has optimum ray trajectories shown in Fig. 2. To account crudely for finite beam divergence, we model a Gaussian beam emerging from a corrugated waveguide with i.d. of $9 \mathrm{~cm}$ by means of three rays. All rays have the same $\phi_{\ell}$, but the outer rays have $\theta_{\ell}$ that differ by $\pm 1^{\circ}$ from the central ray. For an optimal $f=148 \mathrm{GHz}$, the maximum $d I_{d} / d \psi$ at $\psi=0.7$ occurs if the central ray is launched 


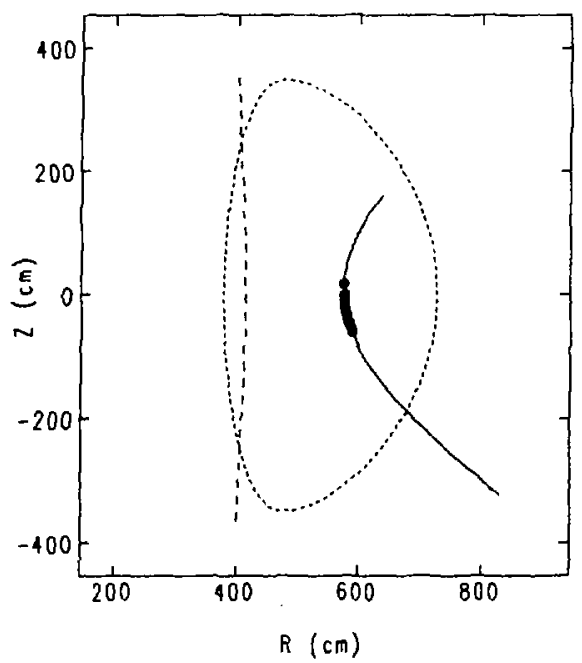

Fig. 1. Poloidal projection of a ray trajectory launched fat below the equatorial plane and passing up through the plasma center. The short-dash curve is the $\psi=0.95$ flux contour of an equilibrium calculated for the JTER tokamak. The long-dash curve is the fundamental cyclotron resonance. The filled circles indicate the location of wave absorption.

with $\phi_{?}=45.5^{\circ}$ and $\theta_{\ell}=23^{\circ}$. At the absorption peak, $N_{\|} \approx 0.5$, slightly more than the 0.4 found for equatorial jaunch. The total driven current is $0.030 \mathrm{~A} / \mathrm{W}$, which corresponds to $\eta=0.12 \times 10^{20} \mathrm{~A} / \mathrm{Wm}^{2}$. The middle $80 \%$ of the current occurs in a width $\Delta \psi=0.03$. Thus, the efficiency and especially the localization are significantly better for the alternative launch geometry than for equatorial launch.

\section{CONCLUSIONS}

Central current drive, optimized for either equatorial or bottom launch, is found to have an efficiency of $\eta \approx 0.2 \times 10^{20} \mathrm{~A} / \mathrm{Wm}^{2}$. Localization is somewhat better with equatorial launch.

Current drive just inside the $q=\mathbf{2}$ surface is more efficient and much more localized if the power is launched so that the power is absorbed while the microwave beam is nearly parallel to the flux surface. A specifie launcis geornetsy with these characteristics is found here for ITER. Further studies sthould address the degradation of efficiency and localization that would occur if $T_{e}$ were lower at $q=2$ or if scattering by drif-wave fluctuations caused the beam to broaden significantly.

For all of the current-drive schemes discussed here, we must assass the prospects for tracking moving flux surfaces using the simplest possible technology.

\section{ACKNOWLEDGMENTS}

Many people aided the author in the performence of this work. R. E. Bulmer provided 


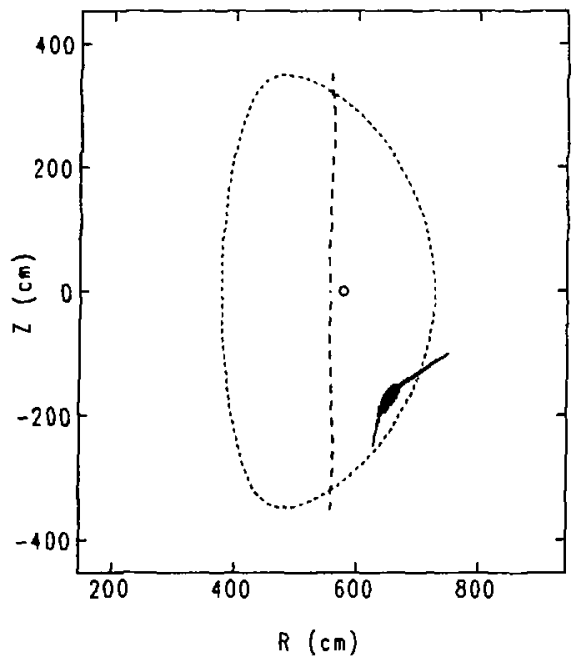

Fig. 2. Poloidal projection of three ray trajectories launched below the equatorial plane at angles designed to drive current localized just inside the $q=2$ surface. The open circle is the magnetic axis.

the equilibrium magnetic-field results. A. V. Kluge, A. H. Kritz, and 1. P. Shkarofsky made major contributions to the ray-trecing code. $R$. H. Cohen made his calculations of current-drive efficiency available.

This work was performed under the auspices of the U.S. Department of Energy by Lawrence Livermore National Laboratory under contract number W-7405-ENG-48.

\section{REFERENCES}

' I. P. Shkarofsky, J. Plasma Phys. 35, 319 (1986); 1. P. Shkarofsky and A. K. Ghosh, Technical Report 232-1, MPB Technologies Inc. (1984).

2 R. H. Cohen, Phys. Fluids 30, 2442 (1987) and 31, 421 (1988).

${ }^{3}$ R. W. Harvey, K. D. Marx, and M. G. McCoy, Nucl. Fusion 21, 153 (1981); C. F. F. Karney and N. J. Fisch, Phys. Fluids 28, 116 (1985).

4 K. Schitikowski, Annals of Operations Research 5,485 (1986).

3 E. Westerhof, Nucl. Fusion 27, 1929 (1987).

6 G. Giruzzi, T. J. Schep, and E. Westerhof, "Current Drive by Electron Cyclotron Waves in NET," presented at Meeting of the European Physical Society (Venice, March 1989). 